\title{
O USO DE ESTRATÉGIAS DE COMUNICAÇÃO EM CUIDADOS PALIATIVOS NO BRASIL: REVISÃO INTEGRATIVA*
}

\author{
Kelviani Ludmila dos Santos Almeidaํ, Dayse Maioli Garcia ${ }^{2}$
}

${ }^{1}$ Enfermeira. Especialista em Enfermagem Clínico-Cirúrgica. Sociedade Beneficente de Senhoras Hospital Sírio-Libanês. São Paulo, SP, Brasil.

${ }^{2}$ Enfermeira. Mestre em Enfermagem. Sociedade Beneficente de Senhoras Hospital Sírio-Libanês. São Paulo, SP, Brasil.

RESUMO: Os cuidados paliativos expressam um campo de cuidados integrais realizado por uma equipe multidisciplinar. A comunicação é uma ferramenta indispensável para essa abordagem. Por meio dela podemos ofertar um adequado controle de sintomas, cuidado individualizado e de qualidade para que conflitos e anseios possam ser resolvidos e a autonomia preservada. O objetivo proposto foi conhecer estratégias de comunicação utilizadas no Brasil para pacientes em cuidados paliativos. Trata-se de uma revisão integrativa, na qual foram levantados artigos em quatro bases de dados e um portal de teses no período de 2009 a 2014. Os artigos relevantes ao tema totalizaram dezenove, destes dezesseis foram utilizados. Os resultados apontaram que as estratégias mais frequentemente citadas foram escuta ativa, silêncio terapêutico, toque afetivo, o ouvir e a empatia, porém, mesmo sendo reconhecidas pela literatura, seu uso ainda é escasso e profissionais necessitam de capacitação emergente para a abordagem paliativa.

DESCRITORES: Cuidados paliativos; Comunicação em saúde; Comunicação interdisciplinar; Neoplasias.

\section{USE OF COMMUNICATION STRATEGIES IN PALLIATIVE CARE IN BRAZIL: INTEGRATIVE REVIEW}

ABSTRACT: Palliative care is a comprehensive care field
served by a multidisciplinary team. Communication is an
essential tool for this approach. Through it an adequate
control of symptoms, as well as individualized and quality
care can be offered, so that conflicts and anxieties can be
solved and autonomy preserved. The proposed objective
was to identify communication strategies used in Brazil
for patients in palliative care. An integrative review was
developed wherein articles were located in four databases
and a portal of theses in the period from 2009 to 2014 . The
articles relevant to the topic totaled 19 , from which 16
were used. The results showed that the most frequently
cited strategies were active listening, therapeutic silence,
and affective touch, hearing, and empathy. However, even
though recognized by the literature, the use of this method
is still rare, and professionals need emerging training in the
palliative approach.

DESCRIPTORS: Palliative care; Health communication; Interdisciplinary communication; Neoplasms.

\section{EL USO DE ESTRATEGIAS DE COMUNICACIÓN EN CUIDADOS PALIATIVOS EN BRASIL: REVISIÓN INTEGRATIVA}

RESUMEN: Los cuidados paliativos representan un campo de cuidados integrales realizado por un equipo multidisciplinar. La comunicación es una herramienta indispensable para ese abordaje. Por medio de ella, podemos ofrecer un control adecuado de síntomas, cuidado individualizado y de cualidad para que conflictos y ansias puedan ser resolvidos y la autonomía preservada. El objetivo propuesto fue conocer estrategias de comunicación utilizadas en Brasil para pacientes en cuidados paliativos. Es una revisión integrativa, en la cual fueron investigados artículos en cuatro bases de datos y un portal de tesis en el periodo de 2009 a 2014. Los artículos relevantes al tema totalizaron diecinueve; de estes dieciseis fueron utilizados. Los resultados apuntan que las estrategias más frecuentemente referidas fueron escucha activa, silencio terapéutico, toque afectivo, el oír y la empatía, pero, a pesar de reconocidas por la literatura, su uso es todavía escaso y profesionales necesitan de capacitación para el abordaje paliativo.

DESCRIPTORES: Cuidados paliativos; Comunicación en salud; Comunicación interdisciplinar; Neoplasias.

\footnotetext{
*Artigo extraído do Trabalho de Conclusão de Curso do Programa de Residência em Enfermagem Clínico-Cirúrgica, intitulado: "O uso de estratégias de comunicação em cuidados paliativos no Brasil: Uma revisão integrativa". Sociedade Beneficente de Senhoras Hospital Sírio-Libanês, 2015.
}

Autor Correspondente:

Kelviani Ludmila dos Santos Almeida

Sociedade Beneficente de Senhoras Hospital Sírio-Libanês

R. Antônio Monteiro de Almeida, 32 - 18408-500 - Itapeva, SP, Brasil

E-mail: kelviani@hotmail.com
Recebido: 16/01/2015

Finalizado: 29/04/2015 


\section{INTRODUÇÃO}

As transformações sociais ocorridas ao longo do tempo, principalmente atribuídas às mudanças demográficas, epidemiológicas e nutricionais, alteraram o modo de vida das pessoas, trazendo diferentes hábitos e carregando consigo direta relação com o adoecimento. Hoje as doenças crônicas não transmissíveis (DCNT) são consideradas epidemias e em muitos países são a principal causa de morte. A Organização Mundial da Saúde (OMS) traz um rol de DCNT e considera que todas necessitam de contínua atenção e estratégias de políticas públicas de saúde para prevenção e controle ${ }^{(1)}$.

Apesardetodooesforçoparaodesenvolvimento dessas estratégias, ainda são muitos os casos de DCNT, o que leva à indispensabilidade de uma abordagem que transcenda o foco na doença e o objetivo da cura, proporcionando ao indivíduo tratamento de forma holística, visando o controle de sintomas e a qualidade de vida ${ }^{(1-2)}$. Cabe aos profissionais de saúde proporcionar a inserção de um cuidado que vise à qualidade de vida e considere a morte como um processo natural. No cuidado paliativo não se busca agir contra a tecnologia, mas sim ponderar sobre a dignidade da vida e da morte ${ }^{(2)}$.

O termo cuidados paliativos foi eleito pela OMS devido à dificuldade de tradução fidedigna do termo Hospice, local destinado para oferecer abrigo aos peregrinos e viajantes ${ }^{(3)}$. Segundo a definição da OMS de 2002,

\footnotetext{
Cuidado Paliativo é uma abordagem que promove a qualidade de vida de pacientes e seus familiares, que enfrentam doenças que ameacem a continuidade da vida, por meio da prevenção e do alívio do sofrimento. Requer identificação precoce, avaliação e tratamento da dor e outros problemas de natureza física, psicossocial e espiritual $^{(4)}$.
}

Alguns países instituíram o serviço voltado para esse cuidado entre 1999 e 2001, entre eles o Brasil $^{(3)}$. O estudo International Observatory on End of Life Care (IOELC), iniciado no ano de 2003 na Universidade de Lancaster, evidenciou a classe de desenvolvimento de serviços de cuidados paliativos em 234 países. Revelou que cerca da metade dos países dispunham desses serviços, apenas $15 \%$ tinham uma política estrutural de oferta e 33\% não apresentavam iniciativa alguma de atividades nesses cuidados ${ }^{(5)}$. No Brasil, ainda necessitamos avançar muito quando se trata de cuidados paliativos. Em um ranking publicado pela revista The Economist em 2010, segundo os critérios escolhidos, o Brasil ficou como o terceiro pior país para se morrer ${ }^{(6)}$.
O código de ética médica traz a diretriz de como tratar paciente e familiar em relação ao diagnóstico e ao prognóstico de sua doença. A literatura mostra, porém, que muitas vezes o médico tem uma postura paternalista para com o paciente, por vezes omitindo fatos, amenizando a gravidade ou apresentando uma comunicação ambivalente, impossibilitando a total compreensão da patologia pelo paciente e sua tomada de decisão(7).

A relação humana é considerada um ponto importante no cuidado em saúde. A comunicação é algo intrínseco ao ser humano e base das relações, tendo um caráter especial nos pacientes em cuidados paliativos. Estudos apontam, entretanto, que a comunicação em cuidados paliativos ainda é realizada de forma falha ${ }^{(8)}$. O Study to Understand Prognoses and Preferences for Outcomes and Risks of Treatments (SUPPORT) foi pioneiro em demonstrar que a comunicação é deficiente e despertou o interesse para outras pesquisas $^{(9)}$.

A complexa relação humana é permeada pela comunicação, que é feita por meio de duas dimensões: a verbal e não verbal. Na comunicação verbal, utilizamos as palavras, sendo capazes de expressar um pensamento, validar algo e clarificar a mensagem. Já a comunicação não verbal é a que qualifica a verbal, são gestos que acompanham o discurso, compreende o tom de voz, o olhar e as expressões faciais, é o que insere emoção à fala ${ }^{(2)}$.

Diversas estratégias de comunicação são citadas na literatura e o uso de técnicas e dessas estratégias é uma medida terapêutica certamente eficaz, porém nem todos os profissionais de saúde sabem utilizá-las. Em uma revisão integrativa realizada por enfermeiras na Inglaterra, foi verificado que as estratégias e habilidades de comunicação utilizadas eram insatisfatórias, agindo diversas vezes como bloqueio para o paciente expor seus sentimentos, e que para o suporte emocional é necessário o uso adequado dessas estratégias ${ }^{(10)}$.

O Quadro 1 demonstra as estratégias estabelecidas na literatura para adequada comunicação em cuidados paliativos ${ }^{(2-3,11)}$.

Levando em consideração a importância da comunicação para a abordagem paliativa, este estudo tem como objetivo levantar evidências na literatura sobre o uso de estratégias de comunicação realizadas no Brasil, pela equipe multiprofissional, para uma comunicação eficaz em cuidado paliativo. 
Quadro 1 - Estratégias para adequada comunicação em cuidados paliativos baseado na literatura pesquisada. São Paulo, SP, Brasil, 2014.

\begin{tabular}{|c|c|}
\hline Verbal & Não verbal \\
\hline $\begin{array}{l}\text { Disponibilizar tempo e verbalizar disponibilidade } \\
\text { para a atenção e cuidado contínuo }{ }^{(2)} \text {. }\end{array}$ & $\begin{array}{l}\text { Atentar para próprias expressões faciais, para a } \\
\text { aparência física (como vestuário) e para a reação que } \\
\text { você provoca no outro }^{(2)} \text {. }\end{array}$ \\
\hline $\begin{array}{l}\text { Estabelecer junto com o paciente metas e planos de } \\
\text { ação para atingi-las }{ }^{(2)} \text {. }\end{array}$ & \multirow{2}{*}{$\begin{array}{l}\text { Atentar para a postura corporal (membros } \\
\text { descruzados e relaxados, orientação do tórax na } \\
\text { direção do outro, sem gestos excessivos ou que } \\
\text { denotem ansiedade }{ }^{(2)} \text {. }\end{array}$} \\
\hline $\begin{array}{l}\text { Estabelecer opções e apontar diferentes pontos de } \\
\text { vista }^{(2)} \text {. }\end{array}$ & \\
\hline $\begin{array}{l}\text { Estimular a verbalização de medos e angústia. Evitar } \\
\text { a conspiração do silêncio (não evitar falar sobre o } \\
\text { assunto doença) })^{(2)} \text {. }\end{array}$ & $\begin{array}{l}\text { Estar atento às "dicas" não verbais expressas pelo } \\
\text { paciente }^{(3)} \text {. }\end{array}$ \\
\hline $\begin{array}{l}\text { Oferecer feedback ao que o outro manifesta e dar } \\
\text { reforço positivo, elogiando as conquistas }{ }^{(2)} \text {. }\end{array}$ & $\begin{array}{l}\text { Estar junto com mais frequência, evitar ruídos que } \\
\text { dispersem a atenção }{ }^{(2)} \text {. }\end{array}$ \\
\hline $\begin{array}{l}\text { Oferecer informações em pequenas doses, sentindo } \\
\text { a receptividade do paciente }{ }^{(3)} \text {. }\end{array}$ & $\begin{array}{l}\text { Identificar emoções e sentimentos nas expressões } \\
\text { faciais }^{(2-3)} \text {. }\end{array}$ \\
\hline Perguntar o que o paciente sabe sobre sua doença ${ }^{(11)}$. & Manter contato visual ${ }^{(2-3,11)}$ \\
\hline $\begin{array}{l}\text { Realizar perguntas abertas para que ele possa se } \\
\text { expressar }{ }^{(11)} \text {. }\end{array}$ & $\begin{array}{l}\text { Manter proximidade física, preferencialmente } \\
\text { sentando-se próximo }{ }^{(2)} \text {. }\end{array}$ \\
\hline $\begin{array}{l}\text { Repetir a informação pausadamente sempre que } \\
\text { necessário }^{(2)} \text {. }\end{array}$ & Escuta ativa. Ouvir reflexivamente ${ }^{(2-3,11)}$ \\
\hline $\begin{array}{l}\text { Repetir as últimas palavras ditas para estimular a } \\
\text { continuar o assunto }^{(2-3)} \text {. }\end{array}$ & Não interromper o paciente ${ }^{(3,11)}$. \\
\hline Utilizar clareza e sinceridade no que foi dito ${ }^{(2-3,11)}$. & $\begin{array}{l}\text { Promover ambiente reservado e tranquilo para uma } \\
\text { conversa }^{(2)} \text {. }\end{array}$ \\
\hline Utilizar de sinceridade prudente ${ }^{(3,11)}$ & Resposta empática ${ }^{(3)}$ \\
\hline $\begin{array}{l}\text { Utilizar perguntas abertas para estimular a } \\
\text { expressão de sentimentos e valorizar os sentimentos } \\
\text { expressados }^{(11)} \text {. }\end{array}$ & $\begin{array}{l}\text { Respeitar o espaço pessoal do paciente, remover } \\
\text { obstáculos físicos }^{(11)} \text {. }\end{array}$ \\
\hline $\begin{array}{l}\text { Utilizar linguagem coloquial, com vocabulário } \\
\text { adequado à compreensão do outro }{ }^{(2-3)} \text {. }\end{array}$ & Utilizar sorriso $^{(2,11)}$. \\
\hline Validar a compreensão do que foi falado ${ }^{(2-3)}$. & Utilizar silêncio e tom de voz adequado ${ }^{(2)}$. \\
\hline $\begin{array}{l}\text { Verbalizar a disposição para o cuidado e não } \\
\text { abandono. Disposição e aceitação }{ }^{(2,11)} \text {. }\end{array}$ & \multirow[t]{2}{*}{ Utilizar toque afetivo ${ }^{(2,11)}$} \\
\hline $\begin{array}{l}\text { Valorizar todas as informações dadas pelo paciente e } \\
\text { pelo familiar }{ }^{(2)} \text {. }\end{array}$ & \\
\hline
\end{tabular}

\section{METODOLOGIA}

O presente estudo utiliza como método a revisão integrativa da literatura, a qual tem como finalidade reunir e resumir o conhecimento científico já produzido sobre o tema investigado, ou seja, permite buscar, avaliar e sintetizar as evidências disponíveis para contribuir com o desenvolvimento do conhecimento na temática. Para a elaboração da revisão integrativa, seguiram-se os seguintes passos: formulação do tema norteador, construção do banco de dados, estabelecimento dos critérios de inclusão e exclusão, avaliação dos estudos, interpretação e avaliação dos resultados, e por fim apresentação da revisão ${ }^{(12)}$.
Para orientar o estudo, foi elaborada a seguinte questão: Quais são as estratégias de comunicação em cuidados paliativos utilizadas pela equipe multiprofissional no Brasil?

Para a estratégia de busca, utilizou-se os recursos informacionais CINAHAL (Cumulative Index to Nursing and Allied Health Literature), LILACS (Literatura Latino-Americana e do Caribe em Ciências da Saúde), o portal PubMed (National Library of Medicine), que alberga a MEDLINE (Medical Literature Analisys and Retrieval System Online), além da busca realizada no banco de teses da Coordenação de Aperfeiçoamento de Pessoal de Nível Superior (CAPES). Optouse por estas bases de dados e biblioteca por 
entender que atingem a literatura publicada nos países da América Latina e Caribe, como também referências técnico-científicas brasileiras em enfermagem, e incluem periódicos conceituados da área da saúde.

Utilizou-se como descritores do DeCS (Descritores em Ciência da Saúde) e do MeSH (Medical Subject Headings): Palliative Care, Communication, Patient Satisfaction, Nonverbal Communication, Communication Barriers, Interdisciplinary Communication, Neoplasms, Critical IIIness, Terminally III, em versões inglês e português a depender da base de dados utilizada, em diferentes combinações, para a verificação do título, resumo ou assunto. A palavra neoplasm foi usada, pois os cuidados paliativos tem se apresentado em muitas pesquisas ligadas às neoplasias. A busca ocorreu no período de maio a julho de 2014.

Os critérios de inclusão foram artigos publicados no período de 2009 a 2014, em português, inglês ou espanhol, com a população de adultos acima de 19 anos, publicados em periódicos científicos nacionais e internacionais que abordem o Brasil como território.

Foram excluídos estudos duplicados, resumos não disponíveis nas bases de dados, ou textos cujo link apresentava erro, e os estudos com população infantil. Com o propósito de facilitar a análise dos conteúdos, todos os artigos foram lidos e categorizados a partir de um instrumento de coleta de dados elaborado pelo autor, no qual foram incluídas as informações de cada estudo selecionado com a finalidade de sintetizar e agrupar as informações, sendo extraídas dos artigos as seguintes variáveis: título, autor, formação dos autores, ano de publicação, periódico, objetivo, tipo de estudo e conclusão.

\section{RESULTADOS}

Foram identificados 229 estudos nas bases de dados e no portal, dos quais quinze encontravamse repetidos. A seleção por título e resumo resultou em dezenove referências. A análise integral destas levou à exclusão de três estudos, uma vez que não possuíam aderência à temática. Por fim, foram incluídas nesta revisão dezesseis publicações.

Em relação ao ano de publicação, 2013 evidenciou mais estudos, correspondendo a $32 \%$, e 2010 e 2014 apresentaram o menor número de artigos, com 19\%. Os resultados salientam que todos os dezesseis artigos trouxeram alguma estratégia utilizada, doze evidenciaram a necessidade de educação acerca do assunto; dois, além de abordarem a comunicação com o paciente, também falam da comunicação entre a equipe. O Quadro 2 traz a síntese dos estudos incluídos nesta revisão.

Quadro 2 - Descrição dos artigos incluídos na revisão. São Paulo, SP, Brasil, 2014. (parte 1)

\begin{tabular}{|l|l|l|}
\hline Título do artigo & Autor/Ano & Tipo de estudo/ Resultados. \\
\hline $\begin{array}{l}\text { A comunicação na transição } \\
\text { para os cuidados Paliativos: } \\
\text { artigo de revisão. }\end{array}$ & $\begin{array}{l}\text { Borges MM, Santos } \\
\text { Jr R. 2012 }\end{array}$ & $\begin{array}{l}\text { Revisão bibliográfica. A comunicação foi apresentada } \\
\text { como um meio para conquista e manutenção de } \\
\text { um relacionamento mais sólido e saudável entre } \\
\text { equipe, paciente e família. Analisa artigos que trazem } \\
\text { a necessidade de treinamento de habilidades de } \\
\text { comunicação. }\end{array}$ \\
\hline $\begin{array}{l}\text { Concepções da equipe } \\
\text { multiprofissional sobre a } \\
\text { implementação dos cuidados } \\
\text { paliativos na unidade de } \\
\text { terapia intensiva. }\end{array}$ & $\begin{array}{l}\text { Silva CF, Souza DM, } \\
\text { Pedreira LC, Santos } \\
\text { MR, Faustino TN. } \\
2013^{(14)} .\end{array}$ & $\begin{array}{l}\text { Estudo de campo, exploratório descritivo, com } \\
\text { abordagem qualitativa. Ressalta como obstáculo no } \\
\text { desenvolvimento dos cuidados paliativos a falta de } \\
\text { comunicação adequada entre a equipe. }\end{array}$ \\
\hline $\begin{array}{l}\text { Cuidados paliativos na } \\
\text { assistência hospitalar: a } \\
\text { vivência de uma equipe } \\
\text { multiprofissional. }\end{array}$ & $\begin{array}{l}\text { Cardoso DH, } \\
\text { Muniz RM, } \\
\text { Schwartz E, Arrieira } \\
\text { ICO 2013 }{ }^{(15)} .\end{array}$ & $\begin{array}{l}\text { Qualitativo, exploratório e descritivo. Identifica como } \\
\text { desafio a necessidade de qualificar a comunicação e o } \\
\text { trabalho em equipe. }\end{array}$ \\
\hline $\begin{array}{l}\text { Percepção dos enfermeiros } \\
\text { sobre o significado dos } \\
\text { cuidados paliativos em } \\
\text { pacientes com câncer } \\
\text { terminal. }\end{array}$ & $\begin{array}{l}\text { Fernandes MA, } \\
\text { Evangelista CB, } \\
\text { Platel ICS, Agra G, } \\
\text { Lopes MS, } \\
\text { Rodrigues FA. } \\
\text { 2013 }{ }^{(16)} \text {. }\end{array}$ & $\begin{array}{l}\text { Estudo exploratório com abordagem qualitativa. } \\
\text { Ressalta o processo de comunicação como ferramenta } \\
\text { essencial e eficaz para o cuidado do paciente sob a } \\
\text { ótica paliativa. }\end{array}$ \\
\hline \multicolumn{2}{|l}{}
\end{tabular}




\begin{tabular}{|c|c|c|}
\hline Título do artigo & Autor/Ano & Tipo de estudo/ Resultados. \\
\hline $\begin{array}{l}\text { Autonomia em cuidados } \\
\text { paliativos: conceitos e } \\
\text { percepções de uma equipe de } \\
\text { saúde. }\end{array}$ & $\begin{array}{l}\text { Oliveira AC, } \\
\text { Silva MJP. } 2010^{(17)}\end{array}$ & $\begin{array}{l}\text { Exploratório descritivo com abordagem qualitativa. } \\
\text { Sugere que comunicação verbal e não verbais são } \\
\text { elementos indispensáveis para a autonomia do } \\
\text { paciente. }\end{array}$ \\
\hline $\begin{array}{l}\text { Cuidados paliativos para } \\
\text { idosos na unidade de terapia } \\
\text { intensiva: revisão sistemática. }\end{array}$ & $\begin{array}{l}\text { Fonseca AC, } \\
\text { Mendes Jr WV, } \\
\text { Fonseca MJM. } \\
2012^{(18)} \text {. }\end{array}$ & $\begin{array}{l}\text { Revisão sistemática. Traz a comunicação entre a equipe } \\
\text { como questão sobressalente no trabalho. }\end{array}$ \\
\hline $\begin{array}{l}\text { Cuidados paliativos: a } \\
\text { comunicação como estratégia } \\
\text { de cuidado para o paciente } \\
\text { em fase terminal. }\end{array}$ & $\begin{array}{l}\text { Andrade CG, Costa } \\
\text { SFG, Lopes MEL. } \\
2013^{(19)} \text {. }\end{array}$ & $\begin{array}{l}\text { Exploratório, de natureza qualitativa. A pesquisa } \\
\text { evidenciou a importância da comunicação como } \\
\text { estratégia fundamental para respaldo da prática clínica } \\
\text { do enfermeiro. }\end{array}$ \\
\hline $\begin{array}{l}\text { Cuidados paliativos na } \\
\text { formação do profissional da } \\
\text { área de saúde. }\end{array}$ & $\begin{array}{l}\text { Fonseca A, } \\
\text { Geovanini F. } \\
2013^{(20)} \text {. }\end{array}$ & $\begin{array}{l}\text { Ausculta exploratória e informal por meio da } \\
\text { aplicação de um questionário. Tem a comunicação } \\
\text { como importante pilar em cuidados paliativos, com } \\
\text { necessidade de desenvolvimento e aprimoramento de } \\
\text { habilidades de comunicação. }\end{array}$ \\
\hline $\begin{array}{l}\text { Comunicação terapêutica na } \\
\text { enfermagem: dificuldades } \\
\text { para o cuidar de idosos com } \\
\text { câncer. }\end{array}$ & $\begin{array}{l}\text { Petersoni AA, } \\
\text { Carvalhol EC. } \\
2011^{(21)} \text {. }\end{array}$ & $\begin{array}{l}\text { Estudo descritivo. Sugere a capacitação e o incentivo } \\
\text { ao desenvolvimento de habilidades de comunicação } \\
\text { com o objetivo de melhora nos cuidados prestados. }\end{array}$ \\
\hline $\begin{array}{l}\text { O médico frente a novidades } \\
\text { no tratamento do câncer: } \\
\text { quando parar? }\end{array}$ & $\begin{array}{l}\text { Hanna SA, } \\
\text { Marta GN, } \\
\text { Santos FS. } 2011^{(22)} \text {. }\end{array}$ & $\begin{array}{l}\text { Revisão da literatura. Conclui que a não comunicação } \\
\text { médico-paciente é algo presente, o que gera uma } \\
\text { relação frágil, baseada na desconfiança. Traz como } \\
\text { estratégia o ouvir. }\end{array}$ \\
\hline $\begin{array}{l}\text { Estratégias de comunicação } \\
\text { utilizadas por profissionais de } \\
\text { saúde na atenção à pacientes } \\
\text { sob cuidados paliativos. }\end{array}$ & $\begin{array}{l}\text { Araújo MMT, } \\
\text { Silva MJP. } 2012^{(23)} \text {. }\end{array}$ & $\begin{array}{l}\text { Estudo de campo, multicêntrico, descritivo, } \\
\text { exploratório e transversal, com abordagem } \\
\text { quantitativa. Conclui que os profissionais } \\
\text { demonstraram pouco conhecimento acerca das } \\
\text { estratégias comunicacionais. }\end{array}$ \\
\hline $\begin{array}{l}\text { O conhecimento de } \\
\text { estratégias de comunicação } \\
\text { no atendimento à dimensão } \\
\text { emocional em cuidados } \\
\text { paliativos. }\end{array}$ & $\begin{array}{l}\text { Araújo MMT, } \\
\text { Silva MJP. } 2012^{(24)}\end{array}$ & $\begin{array}{l}\text { Estudo descritivo, exploratório, transversal e de } \\
\text { campo, com abordagem quantitativa. Conclui que } \\
\text { o conhecimento dos profissionais de saúde no } \\
\text { que se refere ao suporte emocional de pacientes é } \\
\text { insatisfatório. }\end{array}$ \\
\hline $\begin{array}{l}\text { Questões éticas referentes às } \\
\text { preferências do paciente em } \\
\text { cuidados paliativos. }\end{array}$ & $\begin{array}{l}\text { Abreu CBB, } \\
\text { Fortes PAC. } 2014^{(25)} \text {. }\end{array}$ & $\begin{array}{l}\text { Pesquisa exploratória, de abordagem qualitativa. } \\
\text { Reconhece a comunicação como elemento necessário } \\
\text { para a relação terapêutica, sendo fundamental para o } \\
\text { exercício da autonomia do paciente. }\end{array}$ \\
\hline $\begin{array}{l}\text { Ressignificando o cuidado em } \\
\text { uma unidade especializada } \\
\text { em cuidados paliativos: uma } \\
\text { realidade possível? }\end{array}$ & $\begin{array}{l}\text { Vargas MAO, } \\
\text { Vivan J, Vieira, RW, } \\
\text { Mancia R, } \\
\text { Ramos FRS, } \\
\text { Ferrazzo S, et al. } \\
2013^{(27)} \text {. } \\
\end{array}$ & $\begin{array}{l}\text { Pesquisa qualitativa, estudo de caso único } \\
\text { institucional. Constatou a possibilidade da oferta de } \\
\text { um serviço qualificado em uma unidade de cuidados } \\
\text { paliativos, proporcionando uma morte digna. Destaca } \\
\text { entre esses serviços a comunicação. }\end{array}$ \\
\hline $\begin{array}{l}\text { A música na terminalidade } \\
\text { humana: concepções dos } \\
\text { familiares. }\end{array}$ & $\begin{array}{l}\text { Sales CA, Silva VA, } \\
\text { Pilge RC, Marcon } \\
\text { SS. } 2011^{(28)} \text {. }\end{array}$ & $\begin{array}{l}\text { Estudo de múltiplos casos, qualitativo. Traz a música } \\
\text { como estratégia de comunicação que facilita a } \\
\text { relação interpessoal e potencializa a expressão de } \\
\text { sentimentos. }\end{array}$ \\
\hline $\begin{array}{l}\text { O trabalho noturno da } \\
\text { enfermagem no cuidado } \\
\text { paliativo oncológico. }\end{array}$ & $\begin{array}{l}\text { Silva MM, Moreira } \\
\text { MC, Leite JL, } \\
\text { Erdmann AL. } \\
2013^{(29)} \text {. }\end{array}$ & $\begin{array}{l}\text { Estudo exploratório com abordagem qualitativa. } \\
\text { Sugere que é necessário o desenvolvimento de aptidão } \\
\text { para comunicação e escuta ativa. }\end{array}$ \\
\hline
\end{tabular}




\section{DISCUSSÃO}

O objetivo deste trabalho foi levantar nos artigos as estratégias comunicacionais utilizadas com pacientes em cuidados paliativos no Brasil. Como resultado, deparamo-nos com uma escassa literatura, talvez justificada pela falta de abordagem do assunto na graduação, o que contribui para o desinteresse de discentes pelo tema $^{(13)}$. Muitos profissionais sequer conhecem a real filosofia desse tipo de cuidado e o relatam como sendo prestado apenas ao paciente em fase final de vida ${ }^{(14-16)}$.

Quando falamos de comunicação, uma gama de assuntos relacionados aparece. A leitura dos artigos proporcionou chegar a algumas conclusões sobre estratégias de comunicação em cuidados paliativos; levantara importância que elas exercem na autonomia do paciente; apresentar os sentimentos dos profissionais relacionados à abordagem paliativa; e apontar a dificuldade na comunicação dos profissionais não apenas com o paciente, mas também entre equipes. Tudo isso pode descaracterizar o cuidado interdisciplinar, e aponta para a necessidade de educação na área ${ }^{(13-}$ 26).

A maioria dos estudos reconhece a comunicação como aspecto fundamental para intermediar as relações, sendo que esta assume elevado valor no contexto paliativo ${ }^{(13,15-17,19-20,23,27)}$. No entanto, foi possível verificar que, quando se trata de pacientes em cuidados paliativos, os profissionais atrelam a comunicação apenas a notícias de cunho negativo, o que a torna de difícil gerenciamento para equipe e paciente ${ }^{(20)}$.

As estratégias de comunicação verbal e não verbal apresentadas no Quadro 1 foram pouco evidenciadas nos artigos levantados, demostrando um uso escasso delas. Alguns estudos revelam que profissionais atuantes em cuidados paliativos sequer têm conhecimento das abordagens existentes ${ }^{(23-24)}$.

Entre as estratégias, a escuta ativa esteve presente em $50 \%$ dos artigos ${ }^{(14,16-17,19-20,24-25,28)}$; o uso terapêutico do silêncio em $31 \%$, sendo o segundo mais citado ${ }^{(19,23-25,27)}$; perguntar ao paciente o que ele sabe sobre sua condição, como ele se sente, estimulá-lo a falar sobre seus sentimentos, estabelecer conversas amenas, toque afetivo, contato visual e o uso da empatia estiveram como a terceira mais citada, aparecendo em $25 \%$ dos

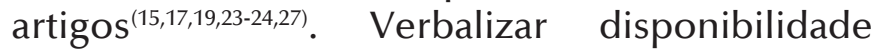
para ajudar, conversar e/ou esclarecer dúvidas, proximidade física/presença e o ouvir aparecem em $19 \%$ dos estudos $115,19,21,23-24,27)$. Em 12,5\% foi encontrado a expressão de sorriso, a realização de gestos positivos, estimular o paciente a falar, respeito do espaço/remover obstáculos, acolher, tom de voz e o uso da sinceridade prudente ${ }^{(15,19,21,23-25)}$. E por fim, presente em $6 \%$ das publicações, perguntar sobre as expectativas do paciente referentes ao tratamento, a clareza, verbalizar compreensão de suas emoções, o meneio positivo coma cabeça, inclinar ocorpo para o paciente, expressão facial, expressar interesse, demonstrar disposição para o cuidado, o uso de perguntas abertas, comunicação compartilhada e música ${ }^{(17,23-24,28)}$. Em 50\% dos estudos levantados se sobressai a escuta ativa. Vale ressaltar que ela não é apenas uma estratégia, e sim um conjunto de sinais verbais e não verbais emitidos pelo profissional como o silêncio, sinais faciais que denotem interesse, o olhar, a aproximação física e o uso de frases curtas para encorajar o prosseguir com a fala ${ }^{(14,16-17,19-20,24-25,28)}$.

Foi relevante a dificuldade dos profissionais de saúde em diferenciar estratégias de comunicação de questões subjetivas como solidariedade, carinho, compaixão, apoio e atenção; por mais que estas denotem sentimentos, não são estratégias estabelecidas $^{(24)}$.

A comunicação é importante para alívio do sofrimento, como elemento terapêutico e diagnóstico, estando vinculada à autonomia do paciente. Um estudo realizado em uma enfermaria de cuidados paliativos, situado em um hospital público, trouxe a eficácia da comunicação compartilhada com o paciente, estando constantemente ligada à capacidade de escolha, domínio próprio e razão, demonstrando que seu benefício é inquestionável ${ }^{(17)}$.

A música também é uma estratégia eficaz de comunicação, contribuindo para um melhor relacionamento interpessoal entre paciente, família e equipe; auxilia na verbalização do paciente, tem finalidade terapêutica, traz alívio da dor, conforto e diminui a ansiedade ${ }^{(28)}$.

Uma pesquisa sobre o trabalho noturno da enfermagem verificou como enfermeiros e técnicos de enfermagem manejam a comunicação nesse período, considerando que a noite para muitos pacientes é associada à morte, e sentimentos como medo, solidão e sofrimento, entre outros, são agravados. O estudo conclui que a comunicação, principalmente a escuta ativa, é elemento essencial para o manejo desses sentimentos, porém reconhecem que, à noite, atender as necessidades do paciente fica 
comprometido, uma vez que os profissionais encontram-se em número reduzido, além da ausência da interdisciplinaridade ${ }^{(29)}$.

Estudos que trouxeram a necessidade de educação dos profissionais para atuarem em cuidado paliativo totalizaram $75 \%$, muitos deles destacando a necessidade de capacitação no que tange à utilização das estratégias de comunicação ${ }^{(13-24)}$.

Tradicionalmente, vemos profissionais de saúde que aprenderam a salvar vidas e agir de forma curativa. Infelizmente não são capacitados suficientemente para atuar com racionalidade terapêutica no controle de sintomas e para considerar a decisão do paciente. Essa falta de conhecimento técnico-científico acerca de terapêuticas para doentes fora da possibilidade de cura levam ao equívoco no tratamento, além de prejudicarem a qualidade de vida e a autonomia do paciente ${ }^{(17,20-22)}$.

Outro vértice dessa falta de conhecimento dos profissionais é o sentimento de frustração, impotência e fracasso quando não conseguem agir de forma curativa, levando-os por vezes a agir com distanciamento do paciente. É importante que os profissionais ponderem sobre suas concepções e sua prática, que reflitam sobre suas competências técnicas e também sobre seus próprios sentimentos diante de um paciente sem possibilidade de cura( ${ }^{(14-15,21)}$.

É fato que a comunicação é uma ferramenta indispensável; por meio dela podemos proporcionar cuidado individualizado e de qualidade para que conflitos e anseios possam ser resolvidos e a autonomia preservada, bem como ofertar o adequado controle de sintomas.

\section{CONSIDERAÇÕES FINAIS}

\section{É possível concluir que estratégias} de comunicação são métodos eficazes e terapêuticos, não sendo intuitivas ou aprendidas empiricamente, e que os profissionais de saúde têm insuficiente ou nenhuma capacitação no que se refere ao uso dessas técnicas.

Associado ao exíguo conhecimento sobre essa temática em cuidados paliativos, a inabilidade de efetivar ações por meio da comunicação é caracterizada como uma barreira para uma assistência de qualidade, que envolva as variadas necessidades do ser humano.

Essa revisão evidenciou que as estratégias comunicacionais são pouco usadas e que por vezes são confundidas com sentimentos, revelando a falta de conhecimento do profissional a respeito do assunto. A partir desse levantamento, é possível afirmar a necessidade de educação dos profissionais acerca da temática "cuidados paliativos" tendo por base o crescente aumento de doenças crônicas. Também, conhecer as estratégias de comunicação é obrigatório quando se busca um cuidado em saúde humanizado e de qualidade. Mostram-se necessárias pesquisas futuras sobre o tema, realizadas por óticas metodológicas variadas.

\section{REFERÊNCIAS}

1. Ministério da Saúde (BR). Secretaria de Vigilância à Saúde. Secretaria de Atenção à Saúde. Diretrizes e recomendações para o cuidado integral de doenças crônicas não transmissíveis: promoção da saúde, vigilância, prevenção e assistência. Brasília, 2008. [internet] Disponível em: http://bvsms.saude.gov.br/ bvs/publicacoes/diretrizes_recomendacoes_cuidado_ doencas_cronicas.pdf. Acesso em: 07 nov. 2014.

2. Matsumoto DY. Cuidados Paliativos: conceito, fundamentos e princípios. In: Carvalho RT, Parsons HA. Manual de cuidados paliativos ANCP. 2 ed. Porto Alegre: Ed Meridional; 2012.

3. Maciel MGS. Definições e princípios. In: Cuidado paliativo. São Paulo: Conselho Regional de Medicina do Estado de São Paulo; 2008. p.15-32.

4. Organização Mundial de Saúde [página da Internet]. Definition of palliative care. Genebra $(\mathrm{CH})$ : OMS, 2002. [acesso 2014 Abr 20]. Disponível em: www.who. int/cancer/palliative/ definition

5. Wright M, Wood J, Lynch T, Clark D. Mapping levels of palliative are development: a global view. Lancaster: International Observatory on End of Life Care Lancaster University; 2006.

6. The Economist Intelligence Unit. The Quality of Death: Ranking end-of-life care across the world. Singapura: LIEN Foundation; The Economist; 2010.

7. Menezes RA. Entre normas e práticas: tomada de decisões no processo saúde/doença. Physis Revista de Saúde Coletiva. Rio de Janeiro 2011; 21(4):1429-1449.

8. Silva MJP. Falando da comunicação. In: Cuidado paliativo. São Paulo: Conselho Regional de Medicina do Estado de São Paulo; 2008. p.33-43.

9. Connors Jr. AF, Dawson NV, Desbiens NA, Fulkerson Jr. WJ, Goldman L, Knaus WA et al. A controlled trial to improve care for seriously III hospitalized patients. The study to understand prognoses and preferences 
for outcomes and risks of treatments (SUPPORT). JAMA. 1995; 274(20):1591-1598.

10. Skilbeck J, Payne S. Emotional support and the role of Clinical Nurse Specialists in palliative care. Journal of Advanced Nursing. 2003; 43(5):521-530.

11. Araújo MMT. Comunicação em cuidados paliativos: proposta educacional para profissionais da saúde. [Tese]. São Paulo: Escola de Enfermagem da Universidade de São Paulo; 2011. 260p.

12. Mendes KDS, Silveira RCCP, Galvão CM. Revisão integrativa: método de pesquisa para incorporação de evidências na saúde e na enfermagem. Texto contexto enferm. 2008 out-dez; 17(4):758-64

13. Borges MM, Santos Jr R. A Comunicação na transição para os cuidados paliativos: artigo de revisão. Revista Brasileira de Educação Médica. 2014; 38(2):275282.

14. Silva CF, Souza DM, Pedreira LC, Santos MR, Faustino TN. Concepções da equipe multiprofissional sobre a implementação dos cuidados paliativos na unidade de terapia intensiva. Ciência \& Saúde Coletiva. 2013; 18(9):2597-2604.

15. Cardoso DH, Muniz RM, Schwartz E, Arrieira ICO. Cuidados paliativos na assistência hospitalar: a vivência de uma equipe multiprofissional. Texto Contexto Enferm. Florianópolis 2013; 22(4):1134-41.

16. Fernandes MA, Evangelista CB, Platel ICS, Agra G, Lopes MS, Rodrigues FA. Percepção dos enfermeiros sobre o significado dos cuidados paliativos em pacientes com câncer terminal. Ciência \& Saúde Coletiva. 2013; 18(9):2589-2596.

17. Oliveira AC, Silva MJP. Autonomia em cuidados paliativos: conceitos e percepções de uma equipe de saúde. Acta Paul Enferm. 2010; 23(2):212-217.

18. Fonseca ACF, Mendes Jr WV, Fonseca MJM. Cuidados paliativos para idosos na unidade de terapia intensiva: revisão sistemática. Rev Bras Ter Intensiva. 2012; 24(2):197-206.

19. Andrade CG, Costa SFG, Lopes MEL. Cuidados paliativos: a comunicação como estratégia de cuidado para o paciente em fase terminal. Ciência \& Saúde Coletiva. 2013; 18(9):2523-2530.

20. Fonseca A, Geovanini F. Cuidados paliativos na formação do profissional da área de saúde. Revista Brasileira de Educação Médica. 2013; 37(1):120-125.

21. Peterson AA, Carvalho EC. Comunicação terapêutica na enfermagem: dificuldades para o cuidar de idosos com câncer. Rev Bras Enferm. Brasilia 2011; 64(4):692-697.
22. Hanna SA, Marta GN, Santos FS. O médico frente a novidades no tratamento do câncer: quando parar? Rev Assoc Med Bras. 2011; 57(5):588-593.

23. Araújo MMT, Silva MJP. Estratégias de comunicação utilizadas por profissionais de saúde na atenção a pacientes sob cuidados paliativos. Rev Esc Enferm USP. 2012; 46(3):626-632.

24. Araújo MMT, Silva MJP. O conhecimento de estratégias de comunicação no atendimento à dimensão emocional em cuidados paliativos. Texto Contexto Enferm. Florianópolis 2012; 21(1):121-129.

25. Abreu CBB, Fortes PAC. Questões éticas referentes às preferências do paciente em cuidados paliativos. Rev Bioét. 2014; 22(2):299-308.

26. Sousa KC, Carpigiani B. Ditos, não ditos e entreditos: a comunicação em cuidados paliativos. Psicologia: Teoria e Prática. 2010; 12(1):97-108.

27. Vargas MAO, Vivan J, Vieira RW, Mancia JR, Ramos FRS, Ferrazzo $S$ et al. Ressignificando o cuidado em uma unidade especializada em cuidados paliativos: uma realidade possível? Texto Contexto Enferm. Florianópolis 2013; 22(3):637-645.

28. Sales CA, Silva VA, Pilger C, Marcon SS. A música na terminalidade humana. Rev Esc Enferm USP. 2011; 45(1):138-145.

29. Silva MM, Moreira MC, Leite JL, Erdmann AL. O trabalho noturno da enfermagem no cuidado paliativo oncológico. Rev Latino-Am Enfermagem. 2013; 21(3):07. 